\title{
Statins and perihemorrhagic edema in patients with spontaneous intracerebral hemorrhage
}

Jens Witsch, MD, Fawaz Al-Mufti, MD, E. Sander Connolly, MD, Sachin Agarwal, MD, MPH, Kara Melmed, MD, David J. Roh, MD, Jan Claassen, MD, PhD, and Soojin Park, MD

Neurology ${ }^{\circledR}$ 2019;92:e2145-e2149. doi:10.1212/WNL.0000000000006931

\section{Abstract}

\section{Objective}

To test the hypothesis that in patients with spontaneous intracerebral hemorrhage (ICH), perihemorrhagic edema to hematoma ratio ( $\mathrm{rPHE}$ ) on admission CT scan $(\mathrm{aCT})$ is unaffected by home statin use when time from symptom onset to $\mathrm{aCT}$ is controlled for.

\section{Methods}

In a single-center prospective cohort of 176 consecutive ICH patients, 2 investigators independently determined hematoma and perihemorrhagic edema (PHE) volumes by using semiautomated validated software. rPHE were dichotomized at the median ratio $(>0.75$ vs $\leq 0.75)$. We used binary logistic regression to test for associations with rPHE.

\section{Results}

In patients using statins as home medication before hospital admission $(n=38)$ compared to patients without prior statin use $(\mathrm{n}=138)$, median PHE volumes were $15.8 \mathrm{~mL}$ (interquartile range [IQR] 6.5-39.4) vs $10.8 \mathrm{~mL}$ (IQR 5.1-26.8), $p=0.2$. rPHE was 0.71 (IQR 0.56-1.0) vs 0.74 (IQR 0.52-1.0), $p=0.79$. In a binary logistic regression model, time of aCT relative to symptom onset (odds ratio [OR] 1.02, confidence interval [CI] 1.01-1.12, $p=0.016$ ) and presence of intraventricular hemorrhage on aCT (OR 0.40, CI $0.20-0.78, p=0.007$ ) were but prior statin use was not (OR 1.17, CI 0.55-2.52, $p=0.68$ ) associated with rPHE.

\section{Conclusion}

Use of statins before hospital admission for $\mathrm{ICH}$ is not associated with reduced rPHE on admission CT. In future studies, imaging timing relative to $\mathrm{ICH}$ onset needs to be controlled for in order to avoid confounding.

\author{
Correspondence \\ Dr. Witsch \\ jensjulianwitsch@gmail.com
}

MORE ONLINE

- CME Course

NPub.org/cmelist

From the Departments of Neurology (J.W., S.A., K.M., D.J.R., J.C., S.P.) and Neurosurgery (E.S.C.), Columbia University Medical Center, New York, NY; Department of Neurology (J.W.), Yale School of Medicine, New Haven, CT; and Departments of Neurology, Neurosurgery, and Radiology (F.A.-M.), Westchester Medical Center, New York Medical College, Valhalla.

Go to Neurology.org/N for full disclosures. Funding information and disclosures deemed relevant by the authors, if any, are provided at the end of the article. 


\section{Glossary}

aCT = admission CT; ICH = intracerebral hemorrhage; IVH = intraventricular hemorrhage; PHE = perihemorrhagic edema; rPHE = perihemorrhagic edema to hematoma ratio.

Relative perihemorrhagic edema (PHE) size (edema to hematoma ratio $[\mathrm{rPHE}])$ on admission $\mathrm{CT}(\mathrm{aCT})$ as well as peak PHE during the hospital course have been associated with functional outcomes at 90 days in patients with intracerebral hemorrhage (ICH). ${ }^{1,2}$ These findings make PHE a candidate target for interventions aiming to improve outcome after ICH. ${ }^{1,3}$ One potential modifier of PHE are statins, which were shown to decrease the extent of PHE on aCT in patients who used them before ICH onset, but this finding was not reproducible across studies. 4,5

Notably, in these studies the symptom onset to aCT time interval was not controlled for. In patients with $\mathrm{ICH}$, the bleed and PHE sizes as well as presence or absence of $\mathrm{ICH}$ associated intraventricular hemorrhage (IVH) on aCT are dependent on CT timing. ${ }^{6,7}$ This study was conducted to test 2 hypotheses: that (1) prior statin use is not associated with rPHE and (2) ICH-onset-to-CT timing drives rPHE on aCT.

\section{Methods}

\section{Patient selection and data collection}

We retrospectively analyzed patient data from a prospective cohort of patients with nontraumatic ICH. Trained study investigators prospectively collected all demographic and clinical data, including outcome variables.

From September 2009 to October 2012, hematoma and edema volumes were routinely measured in all patients admitted during this period. We used the same methodologies regarding data collection and PHE/hematoma measurements as the ones employed in previous studies. ${ }^{3,6}$ Two investigators blinded to clinical variables measured admission hematoma and PHE volumes using MIPAV software package (V.4.3; $\mathrm{NIH}$, Bethesda, MD). We averaged independent observer results. Intraclass correlation coefficients were 0.97 for hematoma volume and 0.88 for PHE volume.

\section{Neuroimaging and semiautomated volumetric measurements}

The assessed radiographic variables included hematoma location and boundaries, PHE boundaries, and presence of IVH. The treating neurointensivists determined the presumed etiology of each ICH by consensus during weekly meetings, based on a combination of demographic/clinical data and radiographic appearance.

\section{Standard protocol approvals and patient consents}

The institutional review board approved the study. Written informed consent was obtained from patients or family members.

\section{Statistical analysis}

We used SPSS software for analyses (version 24; SPSS, Chicago, IL). Data are shown as median and interquartile range or percentages. We used $\chi^{2}$ or Mann-Whitney $U$ tests to assess for differences between groups. First we compared patients who had used statins before their admission as a home medication with those who did not use statins as assessed for each patient as part of our prospective database. We then compared patients with a large edema to hematoma ratio with those with a small ratio. The cutoff was chosen at the median ratio of 0.75 ( $\leq 0.75$ vs $>0.75$, edema volume divided by hematoma volume). Next we entered factors that were different among groups in univariate analyses in a binary logistic regression model to test for associations with rPHE. As a secondary analysis, we stratified the cohort by absolute edema volumes, dichotomized at the median $(>11$ vs $\leq 11$ $\mathrm{mL}$ ). For continuous variables entered in the regression analyses, the following increments were used: time per hour, age per year. We set the statistical significance level at $p<0.05$.

\section{Data availability}

All relevant data are presented within the article and its supporting information files. Additional information can be obtained upon request to the corresponding author.

\section{Results}

Table 1 shows baseline characteristics of ICH patients with $(\mathrm{n}=38)$ and without $(\mathrm{n}=138)$ prior statin use. rPHE was not different between the 2 groups $(p=0.79)$.

\section{Relative edema volumes}

To assess for factors associated with rPHE, we then separated the cohort by rPHE, dichotomized at the median, into patients with large rPHE and those with small rPHE (table 2). Both groups had similar proportions of premorbid statin use $(p=0.90)$.

In a binary logistic regression model, longer time interval between symptom onset and $\mathrm{aCT}$ and absence of IVH on $\mathrm{aCT}$ were associated with larger $\mathrm{rPHE}$, while premorbid statin use was not (table 3 ).

\section{Absolute edema volumes}

As compared to patients with small PHE $(\mathrm{n}=87)$, patients with large PHE $(n=87)$ on aCT had similar proportions of statin users among them ( $21.8 \%$, each group) and larger hematoma volumes (median 38 vs $7.22 \mathrm{~mL}, p \geq 0.001$ ). Patients with large PHE were older ( 70 vs 61 years, $p=0.034)$ and had a higher proportion of lobar bleeds ( $23 \%$ vs $9 \%, p=0.006)$. 
Table 1 Baseline characteristics of patients with intracerebral hemorrhage (ICH), stratified by statin use before admission

\begin{tabular}{|c|c|c|c|}
\hline & Statins $(n=38)$ & No statins $(n=138)$ & $p$ Value \\
\hline Age, y, median (IQR) & $71(63-81)$ & $63(52-78)$ & 0.02 \\
\hline Men, $\mathrm{n}(\%)$ & $73(54)$ & $24(63)$ & 0.3 \\
\hline Caucasian, n (\%) & $16(42.1)$ & $35(25.4)$ & 0.06 \\
\hline \multicolumn{4}{|l|}{ Clinical/CT findings on admission } \\
\hline ICH score, median (IQR) & $2(1-3)$ & $2(1-3)$ & 0.9 \\
\hline \multicolumn{4}{|l|}{ Hemorrhage location, n (\%) } \\
\hline Deep (thalamus/basal ganglia) & $19(50.0)$ & $80(58.0)$ & 0.6 \\
\hline Lobar & $7(18.4)$ & $25(18.1)$ & \\
\hline Infratentorial & $2(5.3)$ & $16(11.6)$ & \\
\hline \multicolumn{4}{|l|}{ ICH etiology, n (\%) } \\
\hline Hypertensive & $19(50.0)$ & $83(60.1)$ & 0.5 \\
\hline Amyloid & $4(10.5)$ & $11(8.0)$ & \\
\hline Other & 15 (39.5) & 44 (31.9) & \\
\hline Onset-to-CT time, h, median (IQR) & $5.3(2.0-9.1)$ & $7.0(1.5-11.2)$ & 0.6 \\
\hline Edema volume, mL, median (IQR) & $15.8(6.5-39.4)$ & $10.8(5.1-26.8)$ & 0.2 \\
\hline Hematoma volume, mL, median (IQR) & $18.7(6.6-65.4)$ & $16.2(7.2-36.0)$ & 0.2 \\
\hline Edema-hematoma ratio, median (IQR) & $0.71(0.56-1.0)$ & $0.74(0.52-1.0)$ & 0.79 \\
\hline Hematoma expansion ( $\geq 33 \%), n(\%)$ & $2(5.3)$ & $25(18.1)$ & 0.05 \\
\hline \multicolumn{4}{|l|}{ Outcomes, median (IQR) } \\
\hline mRS at 3 months & $4(1-6)$ & $4(3-6)$ & 0.31 \\
\hline mRS at 12 months & $3(0-6)$ & $4(1-6)$ & 0.13 \\
\hline
\end{tabular}

Abbreviations: IQR = interquartile range; $\mathrm{mRS}=$ modified Rankin Scale.

aCT timing, presence of IVH on aCT, and rate of hematoma expansion were not different between the groups. In a binary regression model, older age (OR 1.059, CI 1.005-1.116, $p=$ 0.03 ), larger hematoma volume (OR 1.284, CI 1.176-1.402, $p<0.0001$ ), and longer time to aCT (OR 1.118, CI $1.010-1.237, p=0.03$ ) were associated with larger PHE, while prior statin use and hematoma location were not.

\section{Discussion}

In a prospective cohort of patients with spontaneous $\mathrm{ICH}$, we found that the use of statins before admission does not affect the $\mathrm{rPHE}$ on aCT. We furthermore found that a shorter interval between symptom onset and $\mathrm{aCT}$ and the presence of $\mathrm{IVH}$ on aCT were associated with smaller rPHE. These findings confirm our primary null hypothesis as well as our secondary hypothesis.

$\mathrm{PHE}$ in patients with $\mathrm{ICH}$ is a promising treatment target with the potential to improve outcomes. ${ }^{7-10}$ However, many studies do not account for timing of aCT, which may confound results. Choosing a study design in which we controlled for CT timing, we here answered the open question whether statins have a primary prophylactic effect on PHE or not. In a prior study that demonstrated a beneficial effect of statins on PHE, the authors did not control for CT timing and results could not be reproduced since publication. ${ }^{4}$

A prior study has connected peak PHE with clinical outcome at 90 days. ${ }^{2}$ Data on peak PHE were not available in our cohort. Peak PHE is defined as the largest PHE on any CT scan during hospital admission, oftentimes the larger of only 2 volumetric measurements. CT timing relative to symptom onset is usually left to chance. Therefore we do not expect to see an effect of statins on peak PHE. However, this hypothesis needs testing in future studies.

Recent data from the multicenter ERICH cohort showed that statins are associated with improved outcomes in patients with ICH, but it was unclear how this effect was 
Table 2 Baseline characteristics of patients with intracerebral hemorrhage (ICH), stratified by perihemorrhagic edema to hematoma ratio on admission CT scan

\begin{tabular}{|c|c|c|c|}
\hline & $\begin{array}{l}\text { Large PHE/hematoma ratio, } \\
>75 \%(n=84)\end{array}$ & $\begin{array}{l}\text { Small PHE/hematoma ratio, } \\
\leq 75 \%(n=90)\end{array}$ & $p$ Value \\
\hline Age, y, median (IQR) & $71(57.25-79.75)$ & $61.5(51-74.5)$ & 0.06 \\
\hline Men, $\mathbf{n}(\%)$ & $43(51.2)$ & $54(60.0)$ & 0.24 \\
\hline Hematoma location, lobar, n (\%) & $18(21.4)$ & $14(15.6)$ & 0.32 \\
\hline IVH on admission CT, $\mathrm{n}(\%)$ & $46(54.8)$ & $66(73.3)$ & 0.01 \\
\hline Hematoma volume on admission $\mathrm{CT}, \mathrm{mL}$, median (IQR) & $12.31(4.43-32.05)$ & $21.85(11.67-46.0)$ & 0.002 \\
\hline Hematoma expansion, $\mathbf{n}(\%)$ & 10 (11.9) & $16(17.8)$ & 0.28 \\
\hline Prior statin use, $\mathbf{n}(\%)$ & $18(21.4)$ & $20(22.2)$ & 0.90 \\
\hline CT timing, median (IQR) & $8.0(2.17-13.38)$ & $5.0(1.5-9.0)$ & 0.04 \\
\hline \multicolumn{4}{|l|}{ Outcomes, median (IQR) } \\
\hline mRS at 3 months & $3(1-6)$ & $5(3-6)$ & 0.03 \\
\hline $\mathrm{mRS}$ at 12 months & $3(0-6)$ & $4(0-6)$ & 0.18 \\
\hline
\end{tabular}

Abbreviations: IQR = interquartile range; IVH = intraventricular hemorrhage; mRS = modified Rankin Scale; $\mathrm{PHE}=$ perihemorrhagic edema .

mediated. Edema volumes were not available in that study. ${ }^{5}$ In our relatively small patient cohort, we did not find that patients who had been on a statin prior to developing $\mathrm{ICH}$ had better outcomes than those patients who had not been on a statin (table 1). Our findings suggest that, if there is a potentially beneficial effect of statins on outcome in patients with $\mathrm{ICH}$, reduction of $\mathrm{PHE}$ is likely not the mediator. ${ }^{11,12}$

Our finding that rPHE is dependent on CT timing mirrors what is also known to be true for hematoma expansion. ${ }^{6} \mathrm{~A}$ large exploratory study had already suggested the correlation between imaging timing and edema extension, which our study now confirms based on an a priori hypothesis. ${ }^{7}$

Less intuitively understandable is our robust finding that the presence of IVH, which might be interpreted as an indirect sign of advanced ICH expansion, was associated with smaller rPHE. ${ }^{6}$ One may expect that a fully expanded hematoma

Table 3 Binary logistic regression analysis modeling associations with larger hematoma to edema ratio (dichotomized at the median ratio, $>0.75 \mathrm{vs}$ $\leq 0.75$ ) in patients with intracerebral hemorrhage

\begin{tabular}{llll}
\hline Variable & OR & 95\% Cl & $\boldsymbol{p}$ Value \\
\hline Time to admission $\mathrm{CT}, \mathbf{h}$ & 1.016 & $1.012-1.124$ & 0.016 \\
\hline IVH, binary & 0.398 & $0.203-0.780$ & 0.007 \\
\hline Prior statin use & 1.173 & $0.549-2.515$ & 0.678 \\
\hline
\end{tabular}

Abbreviations: $\mathrm{Cl}=$ confidence interval; IVH = intraventricular hemorrhage; $\mathrm{OR}=$ odds ratio might be associated with a larger rPHE, but our data show the opposite. A possible explanation is that most hematomas compared to edema expand earlier relative to ICH onset. ${ }^{6,7}$ Thus IVH could be an indicator of an early and rapid ICH expansion, which may not leave space or time for a proportionally large edema to develop.

In our study, older patients had larger absolute PHE volumes, in line with a recent publication. ${ }^{2}$ It is possible that older age, mediated by relative brain atrophy, facilitates PHE expansion.

Our study has several limitations. First, this is a retrospective analysis of a prospective cohort. Second, PHE volumes were routinely measured only from 2009 to 2012 . Clinical care and guidelines have undergone changes since, which may have affected our results. Third, the cohort was unselected but from a single center and relatively small, limiting the generalizability of our results. To achieve optimal statistical power, we dichotomized edema data at the median, but it is unclear if this is biologically or clinically meaningful, as well. Fourth, type and dosage of statins are not documented in our prospective dataset.

In patients with $\mathrm{ICH}$, statin use before hospital admission is not associated with the extent of PHE, which seems to depend on aCT timing relative to symptom onset and presence or absence of IVH. In future studies on PHE, aCT timing needs to be controlled for, to avoid confounding.

\section{Author contributions}

J. Witsch drafted, revised, and gave final approval for the manuscript and conducted the statistical analysis. F. Al-Mufti provided suggestions for revising the manuscript and gave 
final approval. E.S. Connolly provided suggestions for revising the manuscript and gave final approval. S. Agarwal provided suggestions for revising the manuscript and gave final approval. K. Melmed provided suggestions for revising the manuscript and gave final approval. D.J. Roh provided suggestions for revising the manuscript and gave final approval. J. Claassen provided suggestions for revising the manuscript and gave final approval. S. Park provided project supervision and suggestions for revising the manuscript and gave final approval.

\section{Study funding}

Dr. Witsch received research support from Deutsche Forschungsgemeinschaft (research fellowship Wi 4300/1-1), not targeted to this study.

\section{Disclosure}

The authors report no disclosures relevant to the manuscript. Go to Neurology.org/ $\mathrm{N}$ for full disclosures.

\section{Publication history}

Received by Neurology July 22, 2018. Accepted in final form December 14, 2018.

\section{References}

1. Yang J, Arima H, Wu G, et al. Prognostic significance of perihematomal edema in acute intracerebral hemorrhage: pooled analysis from the Intensive Blood Pressure Reduction in Acute Cerebral Hemorrhage Trial studies. Stroke 2015;46:1009-1013.

2. Volbers B, Giede-Jeppe A, Gerner ST, et al. Peak perihemorrhagic edema correlates with functional outcome in intracerebral hemorrhage. Neurology 2018;90: e1005-e1012.

3. Appelboom G, Bruce SS, Hickman ZL, et al. Volume-dependent effect of perihaematomal oedema on outcome for spontaneous intracerebral haemorrhages. J Neurol Neurosurg Psychiatry 2013;84:488-493.

4. Naval NS, Abdelhak TA, Urrunaga N, Zeballos P, Mirski MA, Carhuapoma JR. An association of prior statin use with decreased perihematomal edema. Neurocrit Care 2008;8:13-18.

5. Siddiqui FM, Langefeld CD, Moomaw CJ, et al. Use of statins and outcomes in intracerebral hemorrhage patients. Stroke 2017;48:2098-2104.

6. Witsch J, Bruce E, Meyers E, et al. Intraventricular hemorrhage expansion in patients with spontaneous intracerebral hemorrhage. Neurology 2015;84:989-994.

7. Wu TY, Sharma G, Strbian D, et al. Natural history of perihematomal edema and impact on outcome after intracerebral hemorrhage. Stroke 2017;48:873-879.

8. Grunwald Z, Beslow LA, Urday S, et al. Perihematomal edema expansion rates and patient outcomes in deep and lobar intracerebral hemorrhage. Neurocrit Care 2017; 26:205-212.

9. Murthy SB, Urday S, Beslow LA, et al. Rate of perihaematomal oedema expansion is associated with poor clinical outcomes in intracerebral haemorrhage. J Neurol Neurosurg Psychiatry 2016;87:1169-1173.

10. Kollmar R, Staykov D, Dörfler A, Schellinger PD, Schwab S, Bardutzky J. Hypothermia reduces perihemorrhagic edema after intracerebral hemorrhage. Stroke 2010; 41:1684-1689.

11. Scheitz JF, MacIsaac RL, Abdul-Rahim AH, et al. Statins and risk of poststroke hemorrhagic complications. Neurology 2016;86:1590-1596.

12. Endres M, Nolte $\mathrm{CH}$, Scheitz JF. Statin treatment in patients with intracerebral hemorrhage. Stroke 2018;49:240-246. 


\section{Neurology}

\section{Statins and perihemorrhagic edema in patients with spontaneous intracerebral hemorrhage}

Jens Witsch, Fawaz Al-Mufti, E. Sander Connolly, et al.

Neurology 2019;92;e2145-e2149 Published Online before print February 6, 2019

DOI 10.1212/WNL.0000000000006931

\section{This information is current as of February 6, 2019}

\section{Updated Information \&} Services

References

Subspecialty Collections

Permissions \& Licensing

Reprints including high resolution figures, can be found at: http://n.neurology.org/content/92/18/e2145.full

This article cites 12 articles, 8 of which you can access for free at: http://n.neurology.org/content/92/18/e2145.full\#ref-list-1

This article, along with others on similar topics, appears in the following collection(s): All CBMRT/Null Hypothesis

http://n.neurology.org/cgi/collection/all_cbmrt_null_hypothesis

Information about reproducing this article in parts (figures,tables) or in its entirety can be found online at:

http://www.neurology.org/about/about_the_journal\#permissions

Information about ordering reprints can be found online:

http://n.neurology.org/subscribers/advertise

Neurology ${ }^{\circledR}$ is the official journal of the American Academy of Neurology. Published continuously since 1951, it is now a weekly with 48 issues per year. Copyright () 2019 American Academy of Neurology. All rights reserved. Print ISSN: 0028-3878. Online ISSN: 1526-632X.

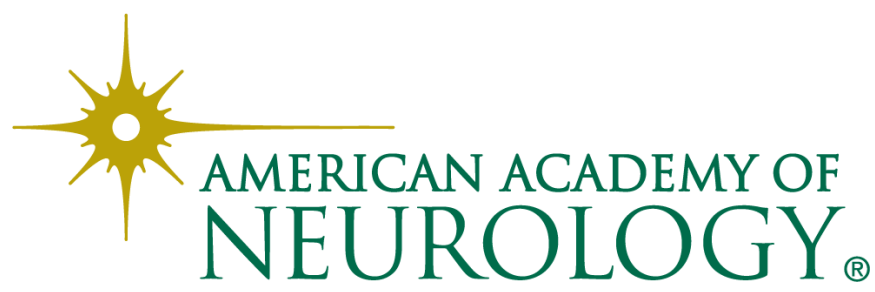

